\title{
CFD Approaches for Modeling Gas-Solids Multiphase Flows - A Review
}

\author{
W.K. Hiromi Ariyaratne ${ }^{1} \quad$ E.V.P.J. Manjula ${ }^{1} \quad$ Chandana Ratnayake $^{2} \quad$ Morten C. Melaaen $^{1}$ \\ ${ }^{1}$ Faculty of Technology, Natural Sciences and Maritime Sciences, University College of Southeast Norway, Post box 235 , \\ N-3603 Kongsberg, Norway, \\ hiromi.ariyaratne, jagath.m.edirisinghe, morten.c.melaae @usn.no \\ 2 Department of POSTEC, Tel-Tek, Kjølnes ring 30, N-3918, Porsgrunn, Norway, chandana. ratnayake@te ${ }^{-}$ \\ tek.no
}

\begin{abstract}
This review study focuses on the application of Computational Fluid Dynamics (CFD) in the investigation of gas-solids multiphase flow systems. The applicability and limitations of conventional models and recent developments of existing multiphase models for the prediction of gas-solids flows are thoroughly overviewed. Use of conventional EulerianEulerian model for granular flows and Lagrangian approach incorporated with Discrete Element Method (CFD-DEM) are quite well proven, however some limitations restrict the use of these models in wide range of applications. Therefore, some new models have been introduced to model gas-solids flows, as example Dense Discrete Phase Model incorporated with Kinetic Theory of Granular Flow (DDPMKTGF), Dense Discrete Phase Model incorporated with Discrete Element Method (DDPM-DEM) and Computational Particle Fluid Dynamics (CPFD) numerical scheme incorporated with the MultiPhaseParticle-In-Cell (MP-PIC) method. These models have been validated for certain applications under certain conditions, however, further validation of these models is still a necessity.
\end{abstract}

Keywords: models, CFD-DEM, DDPM-KTGF, DDPM-DEM, MP-PIC

\section{Introduction}

Applications involving gas-solids multiphase flows are very common in numerous industrial processes and also in various natural phenomena, such as sand storms and cosmic dusts (Li et al., 2012). Pneumatic conveying units, hoppers, solids separation units such as cyclones, bubbling and circulating fluidized beds used in gasification, carbon capture, etc. can be identified as some of the industrial process units involved in gas-solids flows. To optimize the design and operation of industrial processes and also to understand natural phenomena which involve gassolids flows, a thorough understanding of gas-solids flows is needed.
Achievement of this understanding involves the development of experimental measurement techniques, experimentally verified multiphase flow equations and numerical simulation tools (Arastoopour, 2001). Significant effort has been devoted to improving numerical tools, such as Computational Fluid Dynamics (CFD) tool, to predict such complex flows. However, it has been identified that systems containing one or more particulate phases are the most complex and challenging in the field of multiphase flow modeling. To accurately predict the solids behavior, it is necessary to choose a numerical method capable of accounting not only particle-fluid interactions but also for particle-wall and particle-particle interactions in three dimensions and across any particle size distribution (Parker et al., 2013).

Different types of CFD models are available for the prediction of gas-solids flows. Each model has inherent merits and disadvantages. Therefore, a certain model can be appropriate over another depending on the factors prioritized by the user e.g. accuracy of the results, computational time, applicability in large-scale systems, etc. Moreover, the models are still far from perfect and the available models are undergone many improvements within the time. In this review paper, some modeling approaches available for the modeling of gas-solids flow systems are analyzed including their applications and limitations. First, an overview of the models is presented. Then, the two basic approaches and the different models available under basic approaches are discussed.

\section{Basic CFD Approaches for Modeling of Gas-Solids Flows}

A brief summary of the discussed approaches and models are presented in Figure 1. In dealing with modeling of gas-solids flows, the Eulerian-Eulerian and the Eulerian-Lagrangian methods are the frequently used approaches (Chen and Wang, 2014). In Eulerian-Eulerian approach, all the phases are treated as continuous phases while in Eulerian- 


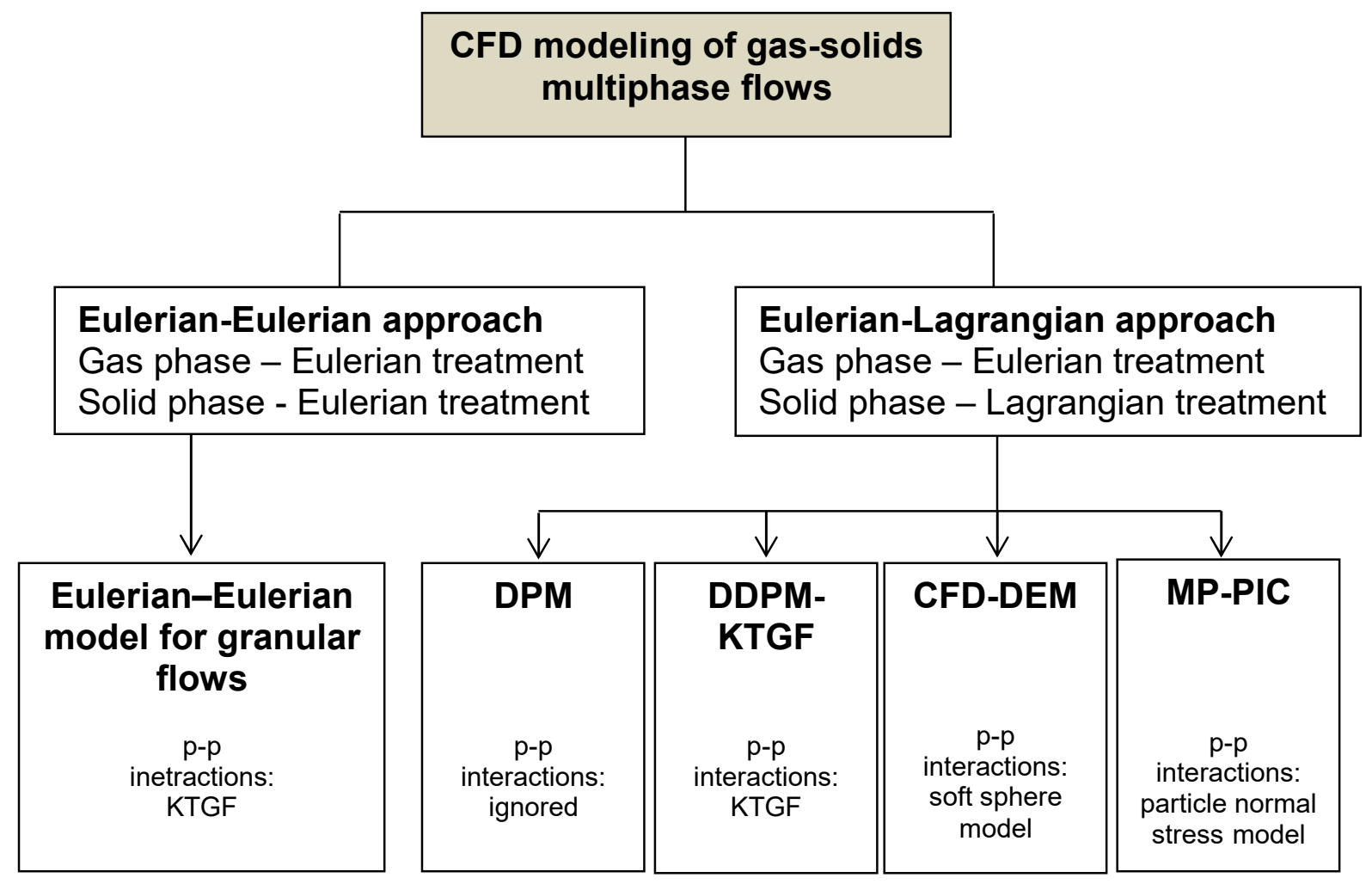

Figure 1. Summary of model approaches for gas-solids multiphase flow modelling.

Lagrangian approach, the fluid phase is treated as a continuous phase, but the solid phase is treated as a discrete phase. Eulerian-Eulerian model for granular flows is described under Eulerian-Eulerian approach and there are four main models under EulerianLagrangian approach, namely, Lagrangian Discrete Phase Model (DPM), Dense Discrete Phase Model incorporated with Kinetic Theory of Granular Flow (DDPM-KTGF), CFD-Discrete Element Method (CFD-DEM) and Computational Particle Fluid Dynamics (CPFD) numerical scheme incorporated with the MultiPhase-Particle-In-Cell (MP-PIC) method.

In addition to the difference in the way of solid phase treatment, another basic difference of the models under both approaches is, the way of treating particleparticle ( $p-p)$ interactions. DPM neglects the $p-p$ interactions, and other models consider the $\mathrm{p}-\mathrm{p}$ interactions through different approaches such as kinetic theory of granular flow, particle normal stress model, soft sphere model, etc.

Much information about the model approaches are discussed in the following sections.

\section{Eulerian-Eulerian approach}

In the Eulerian-Eulerian approach, fluid and particles both are considered as continuous phases which are fully inter-penetrating (Zhang et al., 2012) i.e. solid phase is treated as a pseudo-fluid (Abbasi et al., 2013).
Volume fractions of phases are assumed to be continuous functions of space and time. Since the volume of a phase cannot be occupied by the other phases, the sum of volume fractions is equal to one. This is the concept of the phasic volume fraction (Abbasi et al., 2011). The conservation equations of mass, momentum and energy for the phases are then obtained through an appropriate averaging process (typically ensemble-averaging) (Chen and Wang, 2014). The averaging procedure leads to many unclosed terms, which must be modeled (Snider et al., 2011). Constitutive relationships that are obtained from empirical information and/or kinetic theory are used for this purpose (Abbasi et al., 2013). EulerianEulerian model for granular flows (Euler-granular model) is an example for Eulerian-Eulerian approach. As mentioned (Garg et al., 2012), commercially available codes like ANSYS Fluent, and open source codes like CFDlib, OpenFOAM ${ }^{\circledR}$ and MFiX are all capable of performing Eulerian-Eulerian simulations. Similar forms of governing equations are solved in all these codes and main difference can be found in closures for various sub models (such as solids stresses, interphase drag, etc.) and in numerical treatment.

The Eulerian-Eulerian approach normally requires less computational resources compared to EulerianLagrangian approaches (Chen and Wang, 2014). And this approach is quite traditional and has played a very 
important role in determining the fluid dynamic characteristics of gas-solids flow (Chen and Wang, 2014). Therefore, Eulerian-Eulerian approach has a wide application in gas-solids flows (Weber et al., 2013). However, this approach has major limitations in considering variations of particle properties, as example, wide particle size distribution, density diversification and sphericity consideration. Nevertheless, the particle size differences and/or density variations can affect the gas-solids flow behaviors such as solid segregation (Wang et al., 2014a), hence cannot be neglected in certain situations. In that case, many separate continuity and momentum equations are required to accurately represent the different particle types and sizes in this model (Andrews and O'Rourke, 1996). However, the computational cost of inclusion of many phases cannot then be overlooked, in fact it depends on the computational capacity available. Some researchers have quoted that the Euler-granular model cannot easily account for some characteristics of realistic particles such as shear stresses and inter-particle cohesive forces for Geldart A particles when treated as a pseudo fluid (Chen et al., 2013). Moreover, many researchers emphasize that incorporating of dissipation in the Kinetic Theory of Granular Flow (KTGF) model considering the effects of wall roughness is an important factor for the accurate prediction of results in Eulerian-Eulerian model for granular flows (Chen and Wang, 2014).

\section{Eulerian-Lagrangian Approach}

In Eulerian-Lagrangian approach, the fluid phase is still modeled with time-averaged Navier-Stokes equations and other conservation equations (Yin et al., 2014). The dispersed (solid) phase is treated by tracking a large number of particles through the calculated flow field (Abbasi et al., 2012). Each particle is affected in its trajectory by threedimensional forces and Newtonian equations of motion are used for the calculations (Yin et al., 2014). Commercially available codes like ANSYS Fluent and Barracuda ${ }^{\circledR}$, and open sources codes like MFiX-DEM, KIVA, OpenFOAM ${ }^{\circledR}$ are capable of performing Eulerian-Lagrangian simulations (Garg et al., 2012). The way of treating particle-particle interactions and the numerical method used to solve the equations are the main differences in different Eulerian-Lagrangian codes.

Compared to Eulerian-Eulerian approach, EulerianLagrangian approach can provide analysis of flows with a wide range of particle types, sizes, shapes and velocities ( $\mathrm{Lu}$ et al., 2014). However, if details of particle-particle and particle-wall collisions are explicitly tracked, the traditional Lagrangian models also have some major limitations (Chen and Wang, 2014). For the dense systems in where a large number of particles are involved, the calculation of particleparticle interactions is very complex. It is not possible, even with super computers, to simulate a large-scale system due to the extensive computational cost of tracking each particle (Li et al., 2012). Because of this complexity of calculating particle-particle collisions and the high collision frequency for volume fractions above $5 \%$, these calculations have been limited to the order of $2 \times 10^{5}$ particles and are often restricted to twodimensional solutions without a fluid phase (Snider et al., 2011). To avoid this restriction, some methods have been developed with improvements in calculating particle-particle and particle-wall interactions and also with concept of parcels. The concept of parcel is used to reduce the numbers of particles involved in computations, resulting in a significant acceleration of the speed of simulations (Chen and Wang, 2014). According to (Garg et al., 2012), all publicly available codes except for MFiX-DEM employ a parcel-based approach for the discrete phase. In the parcel approach, a finite number of parcels are tracked rather than using actual individual particles. Each parcel may represent a fractional number of real particles. Typically, several particles with same properties (species, size, density, temperature, etc.) are grouped and put into a parcel. This parcel is also called as a computational/numerical/notional/nominal particle in different literature. However, as ANSYS Fluent mentioned, convergence issues can arise, if fluid volume fraction becomes zero due to either when parcel size is bigger than cell size or too many parcels are squeezed into a cell due to softness of particles. Larger parcel size reduces the number of parcels for a certain mass flow hence lower computational cost. However, the smallest cell should be larger than the largest parcel size (as explained above). Therefore, finding the balance for the optimum mesh is important when using parcel concept. Brief overview of some of Eulerian-Lagrangian models are presented in next sections.

\subsection{Lagrangian Discrete Phase Model (DPM)}

For low and intermediate solids loading, the interparticle spacing is high and hence the negligence of particle-particle interactions might be justifiable. The commercial code; ANSYS Fluent has Lagrangian Discrete Phase Model (DPM) with such a treatment for the flows with solids volume fraction less than $10 \%$. In that model, the volume occupied by solids is not taken into account when assembling the continuous phase equations and particle pressure and viscous stresses due to particles are neglected. The fluid carrier influences the particulate phase via drag and turbulence and if the interaction with continuous phase is enabled, additionally the particles in turn influence the carrier fluid via reduction in mean momentum and turbulence. 
So, this method has either one-way or two-way coupling between the phases, but not four-way coupling where the particle-particle interactions are considered (Fluent, 2013). The particle-wall collisions are modeled through relatively simple models, often based on a simple reflection coefficients of restitution.

\subsection{Dense Discrete Phase Model Incorporated with Kinetic Theory of Granular Flow (DDPM-KTGF)}

Dense Discrete Phase Model incorporated with Kinetic Theory of Granular Flow (DDPM-KTGF) for modeling particle-particle and particle-wall interactions are a quite recently developed model. This model is available in commercial code ANSYS Fluent and open source code OpenFOAM ${ }^{\circledR}$. This is a hybrid model composed with Eulerian-Eulerian and EulerianLagrangian approaches. In low solids volume fractions, the particles are treated in a Lagrangian manner, while in high solids volume fractions, the particles are treated using Eulerian treatment. The solids stress acting on particles resulting from inter-particle interactions is computed from the stress tensor given by the KTGF which is similar to Eulerian-Eulerian approach for granular flows (Euler-granular model). Compared to Lagrangian DPM, this model extends the applicability from dilute to dense phase since this accounts for the effect of volume fraction of solid phase and particleparticle interactions. Still the preciseness of treating particle-particle interactions with KTGF is doubtful. Despite, having benefits of Lagrangian methods and is applicable to large systems, it demands further tests and validations. Some predictions for coal gasification and coal oxy-fuel combustion in circulating fluidized beds (Adamczyk et al., 2014a; Klimanek et al., 2015), circulating fluidized bed boiler (Adamczyk et al., 2014b), impinging particle jet in a channel (Chen and Wang, 2014), solid sorbent carbon capture reactor (Ryan et al., 2013) and ceramic dispersion in liquid pool (Zhang and Nastac, 2014) are made using DDPM-KTGF model. (Ryan et al., 2013) have experienced less stability of DDPM-KTGF solution compared to Euler-granular model and MP-PIC method for a given reactor design and (Chen and Wang, 2014) highlights the requirement of further improvements for DDPM-KTGF model.

\subsection{CFD-Discrete Element Method (CFD- DEM)}

Soft sphere model based on Cundall and Strack, also called "Discrete Element Method (DEM)" or "Distinct Element Method" can be used to explicitly track the particle-particle and particle-wall interaction terms in typical Eulerian-Lagrangian approach (Crowe et al., 2012). This model approach is often referred to as "CFD-DEM" in most of the literature. In-house developed CFD-DEM codes or DEM codes coupled to available CFD platforms through user defined functions are quite common practices. Standalone DEM simulation codes (codes for pure particulate flows without carrier fluid) include open source codes, such as LAMMPS and YADE, and commercial codes, such as EDEM ${ }^{\circledR}$ and ITASCA. Efforts to couple such standalone DEM codes to existing computational fluid dynamic solvers have recently been undertaken. For example, the EDEM code provides users the ability to couple its DEM modules with other CFD codes such as ANSYS Fluent. Recently, OpenFOAM ${ }^{\circledR}$ has been coupled to YADE and LAMMPS (Garg et al., 2012). In DEM, the whole process of collision or contact is solved by numerical integration of the equations of motion. A collision is treated as a continuous process that occurs over a finite time wherein the contact force is calculated as a continuous function of the distance between colliding particles. These are based on physically realistic interaction laws; as example spring, spring dashpot and Coulomb's law of friction. Empirical values for the spring stiffness coefficient, damping constant and friction coefficient are required. Compared to Lagrangian DPM, this model gives more accurate predictions for dense and near-packing limit, however at the cost of slower computations. As many other Eulerian-Lagrangian models, CFD-DEM incorporates with parcel concept in some codes, since recently. The parcel concept reduces an inherent limitation of using DEM in large-scale and dense particle systems. Explicitly tracking collisions of all real particles demands very high computational cost compared to tracking parcels which consist of group of real particles. Billions of real particles in large commercial systems can be analyzed using millions of parcels (Snider, 2007). As example, in-built DEM capability including parcel concept is now available in CFD solver, ANSYS Fluent. It is called Dense Discrete Phase Model incorporated with Discrete Element Method (DDPM-DEM) and this is quite a new feature in ANSYS Fluent. Published data for the application of DDPM-DEM are rare and some information can be found for modeling of micron-particle transport, interactions and deposition in triple lung-airways (Feng and Kleinstreuer, 2014) and coal-direct chemicallooping combustion (Zhang et al., 2014). Another CFD-DEM code; MFiX-DEM is limited to small problem sizes due to high computational cost incurred in the particle neighbor search algorithm in where real particles are considered (Garg et al., 2012). The CFDDEM has been extensively proven to be effective in many gas-solids applications (Chen and Wang, 2014). 


\subsection{Computational Particle Fluid Dynamics (CPFD) Numerical Scheme Incorporated with the Multiphase- Particle-in-Cell (MP-PIC) Method}

The Computational Particle Fluid Dynamics (CPFD) numerical scheme incorporated with the MultiPhaseParticle-In-Cell (MP-PIC) method to describe the solid phase is quite new Eulerian-Lagrangian approach for calculating gas-solids flows. This is a version after several significant improvements of Particle-In-Cell (PIC) method used for single-phase flows since 1960s (Snider, 2001). As Snider, Clark and O'rourke mentioned, the MP-PIC method is, in turn, an extension of the stochastic particle method of the KIVA code (Snider et al., 2011). In the CPFD method, the real particles are grouped into parcels as in many other Eulerian-Lagrangian methods (Zhang et al., 2012). The dynamics of the particle phase is predicted in the MP-PIC method by solving a transport equation which is called Liouville equation for the particle distribution function. The particle distribution function contains particle properties as example, particle spatial location, particle velocity, particle mass, time, etc. (Karimipour and Pugsley, 2012). Unlike DEM models which calculate particle-to-particle force by a springdamper model and direct particle contact, the CPFD methodology models particles' collision force on each particle as a spatial gradient. A particle normal stress model is developed from this concept to describe the particle collisions (Wang et al., 2014b). In the computation, the stress gradient on the grid is first calculated and then interpolated to discrete particles (Abbasi et al., 2013). The model has been undergone through many improvements such as including Bhatnager, Gross and Krook (BKG) collision model for gas/liquid/solids flows (O'Rourke et al., 2009), including collision damping fluctuations due to inelastic collisions (O'Rourke and Snider, 2010), including return-to-isotropy term in collision source term (O'Rourke and Snider, 2012), including the effects of the contact force variations caused by inhibition of relative motions due to different particle sizes and densities (O'Rourke and Snider, 2014), etc. Arena-Flow ${ }^{\circledR}$, Barracuda ${ }^{\circledR}$ and OpenFOAM ${ }^{\circledR}$ are some examples for the software/codes which have CPFD implementation. Compared to Lagrangian DPM, this model can accurately model gas-solids flows of dense and close-pack limits. Solution cost is reduced since the collisions are not directly solved as in DEM and also due to implementation of the parcel concept. Furthermore, MP-PIC method does not need to take the particle collisions implicitly, therefore a much larger time step can be adopted (Yin et al., 2014). As mentioned (Lu et al., 2014), this method can be used to model systems with physical particle counts over $1 \times 10^{15}$ particles. In addition, the CPFD method has shown the ability to model full particle size distribution for any number of solid species and to model particle volume fraction from dilute $(<0.1 \%)$ upto dense $(>60 \%)$. Some of the applications of MP-PIC method are bubbling and circulating fluidized beds (Chen et al., 2013; Jiang et al., 2014; Karimipour and Pugsley, 2012; Lan et al., 2013; Liang et al., 2014; Parker et al., 2013; Wang et al., 2014b; Weber et al., 2013; Yin et al., 2014; Zhang et al., 2012), fluidized bed gasifiers (Abbasi et al., 2011; Loha et al., 2014; Singh et al., 2013; Snider et al., 2011; Thapa et al., 2014), fluidized beds for carbon capture (Breault and Huckaby, 2013; Clark et al., 2013; Parker, 2014; Ryan et al., 2013), gas/liquid/solid fluidized beds (O'Rourke et al., 2009; Vivacqua et al., 2013; Zhao et al., 2009), RayleighTaylor mixing layers (Snider, 2001), sedimentation (Andrews and O'Rourke, 1996; Snider, 2001), downer reactors (Abbasi et al., 2012, 2013), dryer (Bigda, 2014), 3-D particle jet (Snider, 2001), hopper flow (Lu et al., 2014; Snider, 2007), particle flow in U-tube (Snider, 2007).

In addition to these models, Sommerfeld has developed a stochastic collision model to model the inter-particle collisions (Laín and Sommerfeld, 2012). Furthermore, a brief comparison of results obtained using above mentioned models can be found in elsewhere (Chen and Wang, 2014).

\section{Conclusions}

A general overview of some of the available gas-solids flow modeling approaches is made in the current review paper. Eulerian-Eulerian and EulerianLagrangian are the approaches in use. Further, Lagrangian Discrete Phase Model (DPM), Dense Discrete Phase Model incorporated with Kinetic Theory of Granular Flow (DDPM-KTGF), CFDDiscrete Element Method (CFD-DEM) and Computational Particle Fluid Dynamics (CPFD) numerical scheme incorporated with the MultiPhaseParticle-In-Cell (MP-PIC) method are the models discussed under Eulerian-Lagrangian approach.

The conventional Eulerian-Eulerian model for granular flows and CFD-DEM models have widely been used for many applications and validated quite well. Despite this, both models still have major limitations with respect to accuracy and computational cost, hence applying to large scale systems and to model flows with different particle properties are not very straightforward. Therefore, these models are being under improvements and some new models have been introduced to model gas-solids flows, as example DDPM-KTGF, DDPM-DEM and MP-PIC. In addition to getting advantage of Lagrangian treatment of the particles, these models are said to be efficient compared to the conventional models. This might be due to the use of parcel concept and/or due to use of empirical approaches for modeling particle-particle interactions, alternative algorithms and grid. Few 
publications related to use of MP-PIC method are available mainly in fluidized bed applications, however published information for the applications of other models are not very abundant. Therefore, the applicability and validity of these quite recent models for the accurate predictions of gas-solids multiphase flow modeling should be investigated. Moreover, all the models need further improvements in order to apply for wide range of applications and scales.

\section{Acknowledgements}

The authors would like to acknowledge the financial support provided by the Research Council of Norway under PETROMAKS II program and Det Norske oljeselskape ASA.

\section{References}

A. Abbasi, P. E. Ege, and H. I. de Lasa. CPFD simulation of a fast fluidized bed steam coal gasifier feeding section. Chemical Engineering Journal, 174(1): 341-350, 2011. doi:10.1016/j.cej.2011.07.085.

A. Abbasi, M. A. Islam, P. E. Ege, and H. I. de Lasa. Downer reactor flow measurements using CREC-GSOptiprobes. Powder Technology, 224(Supplement C): 111, 2012. doi:10.1016/j.powtec.2012.02.005.

A. Abbasi, M. A. Islam, P. E. Ege, and H. I. de Lasa. CPFD flow pattern simulation in downer reactors. AIChE Journal, 59(5): 1635-1647, 2013. doi:10.1002/aic.13956.

W. P. Adamczyk, P. Kozołub, G. Węcel, A. Klimanek, R. A. Białecki, and T. Czakiert. Modeling oxy-fuel combustion in a 3D circulating fluidized bed using the hybrid EulerLagrange approach. Applied Thermal Engineering, 71(1): 266-275, doi:10.1016/j.applthermaleng.2014.06.063.

2014a.

W. P. Adamczyk, G. Węcel, M. Klajny, P. Kozołub, A. Klimanek, and R. A. Białecki. Modeling of particle transport and combustion phenomena in a large-scale circulating fluidized bed boiler using a hybrid EulerLagrange approach. Particuology, 16(Supplement C): 2940, 2014b. doi:10.1016/j.partic.2013.10.007.

M. J. Andrews and P. J. O'Rourke. The multiphase particlein-cell (MP-PIC) method for dense particulate flows. International Journal of Multiphase Flow, 22(2): 379-402, 1996. doi:10.1016/0301-9322(95)00072-0.

H. Arastoopour. Numerical simulation and experimental analysis of gas/solid flow systems: 1999 Fluor-Daniel Plenary lecture. Powder Technology, 119(2): 59-67, 2001. doi:10.1016/S0032-5910(00)00417-4.

J. Bigda. CPFD Numerical Study of Impact Dryer Performance. Drying Technology, 32(11): 1277-1288, 2014. doi:10.1080/07373937.2014.929586.

R. W. Breault and E. D. Huckaby. Parametric behavior of a $\mathrm{CO}_{2}$ capture process: CFD simulation of solid-sorbent $\mathrm{CO}_{2}$ absorption in a riser reactor. Applied Energy, 112(Supplement $\quad$ C): 224-234, 2013. doi:10.1016/j.apenergy.2013.06.008

C. Chen, J. Werther, S. Heinrich, H.-Y. Qi, and E.-U. Hartge. CPFD simulation of circulating fluidized bed risers.
Powder Technology, 235(Supplement C): 238-247, 2013. doi:10.1016/j.powtec.2012.10.014.

$\mathrm{X}$. Chen and J. Wang. A comparison of two-fluid model, dense discrete particle model and CFD-DEM method for modeling impinging gas-solid flows. Powder Technology, 254(Supplement $\quad$ C): 2014. doi:10.1016/j.powtec.2013.12.056.

S. Clark, D. M. Snider, and J. Spenik. $\mathrm{CO}_{2}$ Adsorption loop experiment with Eulerian-Lagrangian simulation. Powder Technology, 242(Supplement C): 100-107, 2013. doi:10.1016/j.powtec.2013.01.011.

C. T. Crowe, J. D. Schwarzkopf, M. Sommerfeld, and Y. Tsuji. Multiphase flows with droplets and particles, Taylor \& Francis Group, LLC. 2012.

Y. Feng and C. Kleinstreuer. Micron-particle transport, interactions and deposition in triple lung-airway bifurcations using a novel modeling approach. Journal of Aerosol Science, 71(Supplement C): 1-15, 2014. doi:10.1016/j.jaerosci.2014.01.003.

ANSYS fluent theory guide 15.0. Canonsburg, PA, ANSYS Inc. 2013

R. Garg, J. Galvin, T. Li, and S. Pannala. Open-source MFIX-DEM software for gas-solids flows: Part IVerification studies. Powder Technology, 220(Supplement C): 122-137, 2012. doi:10.1016/j.powtec.2011.09.019.

Y. Jiang, G. Qiu, and H. Wang. Modelling and experimental investigation of the full-loop gas-solid flow in a circulating fluidized bed with six cyclone separators. Chemical Engineering Science, 109(Supplement C): 8597, 2014. doi:10.1016/j.ces.2014.01.029.

S. Karimipour and T. Pugsley. Application of the particle in cell approach for the simulation of bubbling fluidized beds of Geldart A particles. Powder Technology, 220(Supplement $\quad$ C): $\quad 63-69, \quad 2012$. doi:10.1016/j.powtec.2011.09.026.

A. Klimanek, W. Adamczyk, A. Katelbach-Woźniak, G. Węcel, and A. Szlęk. Towards a hybrid EulerianLagrangian CFD modeling of coal gasification in a circulating fluidized bed reactor. Fuel, 152(Supplement C): 131-137, 2015. doi:10.1016/j.fuel.2014.10.058.

S. Laín and M. Sommerfeld. Numerical calculation of pneumatic conveying in horizontal channels and pipes: Detailed analysis of conveying behaviour. International Journal of Multiphase Flow, 39(Supplement C): 105-120, 2012. doi:10.1016/j.ijmultiphaseflow.2011.09.006.

X. Lan, X. Shi, Y. Zhang, Y. Wang, C. Xu, and J. Gao. Solids Back-mixing Behavior and Effect of the Mesoscale Structure in CFB Risers. Industrial \& Engineering Chemistry Research, 52(34): 11888-11896, 2013. doi:10.1021/ie3034448.

T. Li, R. Garg, J. Galvin, and S. Pannala. Open-source MFIX-DEM software for gas-solids flows: Part II Validation studies. Powder Technology, 220(Supplement C): 138-150, 2012. doi:10.1016/j.powtec.2011.09.020.

Y. Liang, Y. Zhang, T. Li, and C. Lu. A critical validation study on CPFD model in simulating gas-solid bubbling fluidized beds. Powder Technology, 263(Supplement C): 121-134, 2014. doi:10.1016/j.powtec.2014.05.003.

C. Loha, H. Chattopadhyay, and P. K. Chatterjee. Three dimensional kinetic modeling of fluidized bed biomass 
gasification. Chemical Engineering Science, 109(Supplement $\quad$ C): $\quad$ 53-64, 2014. doi:10.1016/j.ces.2014.01.017.

H. Lu, X. Guo, W. Zhao, X. Gong, and J. Lu. Experimental and CPFD Numerical Study on Hopper Discharge. Industrial \& Engineering Chemistry Research, 53(30): 12160-12169, 2014. doi:10.1021/ie403862f.

P. J. O'Rourke and D. M. Snider. Inclusion of collisional return-to-isotropy in the MP-PIC method. Chemical Engineering Science, 80(Supplement C): 39-54, 2012. doi:10.1016/j.ces.2012.05.047.

P. J. O'Rourke and D. M. Snider. A new blended acceleration model for the particle contact forces induced by an interstitial fluid in dense particle/fluid flows. Powder Technology, 256(Supplement C): 39-51, 2014. doi:10.1016/j.powtec.2014.01.084.

P. J. O'Rourke and D. M. Snider. An improved collision damping time for MP-PIC calculations of dense particle flows with applications to polydisperse sedimenting beds and colliding particle jets. Chemical Engineering Science, 65(22): 6014-6028, 2010. doi:10.1016/j.ces.2010.08.032.

P. J. O'Rourke, P. Zhao, and D. Snider. A model for collisional exchange in gas/liquid/solid fluidized beds. Chemical Engineering Science, 64(8): 1784-1797, 2009. doi:10.1016/j.ces.2008.12.014.

J. Parker, K. LaMarche, W. Chen, K. Williams, H. Stamato, and $\mathrm{S}$. Thibault. CFD simulations for prediction of scaling effects in pharmaceutical fluidized bed processors at three scales. Powder Technology, 235(Supplement C): 115-120, 2013. doi:10.1016/j.powtec.2012.09.021.

J. M. Parker. CFD model for the simulation of chemical looping combustion. Powder Technology, 265(Supplement C): 47-53, 2014. doi:10.1016/j.powtec.2014.01.027.

E. M. Ryan, D. DeCroix, R. Breault, W. Xu, E. D. Huckaby, K. Saha, S. Dartevelle, and X. Sun. Multi-phase CFD modeling of solid sorbent carbon capture system. Powder Technology, 242( Supplement C): 117-134, 2013. doi:10.1016/j.powtec.2013.01.009.

R. I. Singh, A. Brink, and M. Hupa. CFD modeling to study fluidized bed combustion and gasification. Applied Thermal Engineering, 52(2): 585-614, 2013. doi:10.1016/j.applthermaleng.2012.12.017.

D. M. Snider. An Incompressible Three-Dimensional Multiphase Particle-in-Cell Model for Dense Particle Flows. Journal of Computational Physics, 170(2): 523549, 2001. doi:10.1006/jcph.2001.6747.

D. M. Snider. Three fundamental granular flow experiments and CPFD predictions. Powder Technology, 176(1): 36-46, 2007. doi:10.1016/j.powtec.2007.01.032.

D. M. Snider, S. M. Clark, and P. J. O'Rourke. EulerianLagrangian method for three-dimensional thermal reacting flow with application to coal gasifiers. Chemical Engineering Science, 66(6): 1285-1295, 2011. doi:10.1016/j.ces.2010.12.042.

R. Thapa, C. Pfeifer, and B. Halvorsen. Modeling of reaction kinetics in bubbling fluidized bed biomass gasification reactor. Internal Journal of Energy and Environment, 5(1): 35-44, 2014

V. Vivacqua, S. Vashisth, A. Prams, G. Hébrard, N. Epstein, and J. R. Grace. Experimental and CPFD study of axial and radial liquid mixing in water-fluidized beds of two solids exhibiting layer inversion. Chemical Engineering Science, 95(Supplement C): 119-127, 2013. doi:10.1016/j.ces.2013.03.011.

Q. Wang, H. Yang, P. Wang, J. Lu, Q. Liu, H. Zhang, L. Wei, and M. Zhang. Application of CPFD method in the simulation of a circulating fluidized bed with a loop seal Part II-Investigation of solids circulation. Powder Technology, 253(Supplement C): 822-828, 2014a. doi:10.1016/j.powtec.2013.11.040

Q. Wang, H. Yang, P. Wang, J. Lu, Q. Liu, H. Zhang, L. Wei, and M. Zhang. Application of CPFD method in the simulation of a circulating fluidized bed with a loop seal, part I-Determination of modeling parameters. Powder Technology, 253(Supplement C): 814-821, $2014 \mathrm{~b}$. doi:10.1016/j.powtec.2013.11.041.

J. M. Weber, K. J. Layfield, D. T. Van Essendelft, and J. S. Mei. Fluid bed characterization using Electrical Capacitance Volume Tomography (ECVT), compared to CPFD Software's Barracuda. Powder Technology, 250(Supplement C): 138-146, 2013. doi:10.1016/j.powtec.2013.10.005.

S. Yin, W. Zhong, B. Jin, and J. Fan. Modeling on the hydrodynamics of pressurized high-flux circulating fluidized beds (PHFCFBs) by Eulerian-Lagrangian approach. Powder Technology, 259(Supplement C): 52-64, 2014. doi:10.1016/j.powtec.2014.03.059.

D. Zhang and L. Nastac. Numerical modeling of the dispersion of ceramic nanoparticles during ultrasonic processing of aluminum-based nanocomposites. Journal of Materials Research and Technology, 3(4): 296-302, 2014. doi:10.1016/j.jmrt.2014.09.001.

Y. Zhang, X. Lan, and J. Gao. Modeling of gas-solid flow in a CFB riser based on computational particle fluid dynamics. Petroleum Science, 9(4): 535-543, 2012. doi:10.1007/s12182-012-0240-7.

Z. Zhang, L. Zhou, and R. Agarwal. Transient Simulations of Spouted Fluidized Bed for Coal-Direct Chemical Looping Combustion. Energy \& Fuels, 28(2): 1548-1560, 2014. doi:10.1021/ef402521x.

P. Zhao, P. J. O'Rourke, and D. Snider. Three-dimensional simulation of liquid injection, film formation and transport, in fluidized beds. Particuology, 7(5): 337-346, 2009. doi:10.1016/j.partic.2009.07.002. 